\title{
The impact of personality disorder pathology on the effectiveness of cognitive therapy and interpersonal psychotherapy for major depressive disorder
}

\author{
Citation for published version (APA):
}

van Bronswijk, S. C., Lemmens, L. H. J. M., Viechtbauer, W., Huibers, M. J. H., Arntz, A., \& Peeters, F. (2018). The impact of personality disorder pathology on the effectiveness of cognitive therapy and interpersonal psychotherapy for major depressive disorder. Journal of Affective Disorders, 225, 530-538. https://doi.org/10.1016/j.jad.2017.08.043

Document status and date:

Published: 01/01/2018

DOI:

10.1016/j.jad.2017.08.043

Document Version:

Publisher's PDF, also known as Version of record

\section{Document license:}

Taverne

Please check the document version of this publication:

- A submitted manuscript is the version of the article upon submission and before peer-review. There can be important differences between the submitted version and the official published version of record.

People interested in the research are advised to contact the author for the final version of the publication, or visit the DOI to the publisher's website.

- The final author version and the galley proof are versions of the publication after peer review.

- The final published version features the final layout of the paper including the volume, issue and page numbers.

Link to publication

\footnotetext{
General rights rights.

- You may freely distribute the URL identifying the publication in the public portal. please follow below link for the End User Agreement:

www.umlib.nl/taverne-license

Take down policy

If you believe that this document breaches copyright please contact us at:

repository@maastrichtuniversity.nl

providing details and we will investigate your claim.
}

Copyright and moral rights for the publications made accessible in the public portal are retained by the authors and/or other copyright owners and it is a condition of accessing publications that users recognise and abide by the legal requirements associated with these

- Users may download and print one copy of any publication from the public portal for the purpose of private study or research.

- You may not further distribute the material or use it for any profit-making activity or commercial gain

If the publication is distributed under the terms of Article $25 \mathrm{fa}$ of the Dutch Copyright Act, indicated by the "Taverne" license above, 
Research paper

\title{
The impact of personality disorder pathology on the effectiveness of Cognitive Therapy and Interpersonal Psychotherapy for Major Depressive Disorder
}

\author{
Suzanne C. van Bronswijk ${ }^{\mathrm{a}, *}$, Lotte H.J.M. Lemmens ${ }^{\mathrm{b}}$, Wolfgang Viechtbauer ${ }^{\mathrm{a}}$, \\ Marcus J.H. Huibers ${ }^{\mathrm{c}, \mathrm{d}}$, Arnoud Arntz ${ }^{\mathrm{b}, \mathrm{e}}$, Frenk P.M.L. Peeters ${ }^{\mathrm{a}}$ \\ a Department of Psychiatry and Neuropsychology, Faculty of Health, Medicine and Life Sciences, Maastricht University, P.O. Box 616, 6200 MD Maastricht, The \\ Netherlands \\ b Department of Clinical Psychological Science, Faculty of Psychology and Neuroscience, Maastricht University, P.O. Box 616, 6200 MD Maastricht, The Netherlands \\ ' Department of Clinical Psychology, VU University Amsterdam, Van der Boechorststraat 1, 1081 BT Amsterdam, The Netherlands \\ d Department of Psychology, University of Pennsylvania, 3720 Walnut Street, Philadelphia, PA 19104-6241, USA \\ e Department of Clinical Psychology, University of Amsterdam, PO Box 19268, 1000 GG Amsterdam, The Netherlands
}

\section{A R T I C L E I N F O}

\section{Keywords:}

Depression

Personality

Cognitive Therapy

Interpersonal Psychotherapy

Psychotherapy

\begin{abstract}
A B S T R A C T
Background: Despite extensive research, there is no consensus how Personality Disorders (PD) and PD features affect outcome for Major Depressive Disorder (MDD). The present study evaluated the effects of PD (features) on treatment continuation and effectiveness in Cognitive Therapy (CT) and Interpersonal Psychotherapy (IPT) for MDD.

Methods: Depressed outpatients were randomized to CT $(n=72)$ and IPT $(n=74)$. Primary outcome was depression severity measured repeatedly with the Beck Depression Inventory-II (BDI-II) at baseline, three months, at the start of each therapy session, at post-treatment and monthly during five months follow-up.

Results: Comorbid PD and PD features did not affect dropout. Multilevel and Cox regression models indicated no negative effect of PD on BDI-II change and remission rates during treatment and follow-up, irrespective of the treatment received. For both therapies, higher dependent PD features predicted overall lower BDI-II scores during treatment, however this effect did not sustain through follow-up. Cluster A PD features moderated treatment outcome during treatment and follow-up: individuals with high cluster A PD features had greater BDIII reductions over time in CT as compared to IPT.

Limitations: Not all therapists and participants were blind to the assessment of PD (features), and assessments were performed by one rater. Further research must investigate the state and trait dependent changes of PD and MDD over time.

Conclusions: We found no negative impact of PD on the effectiveness and treatment retention of CT and IPT for MDD during treatment and follow-up. If replicated, cluster A PD features can be used to optimize treatment selection.
\end{abstract}

\section{Introduction}

Individuals with major depressive disorder (MDD) often meet criteria for DSM defined co-morbid personality disorders (PD), in particular PD's grouped in cluster C: obsessive compulsive PD, dependent PD and avoidant PD (Friborg et al., 2014). Despite extensive research spanning several decades, results from research into the impact of comorbid PD on the effectiveness of acute phase treatment for MDD is equivocal; results vary from a negative association between the presence of a co-morbid PD and clinical outcome (Hardy et al., 1995;
Newton-Howes et al., 2014; Reich and Vasile, 1993; Sato et al., 1994) to the absence of any difference between outcome in individuals with and without co-morbid PD (De Bolle et al., 2011; Kool et al., 2005; Moradveisi et al., 2013; Mulder, 2002). These inconsistencies across studies are probably best explained by methodological problems. Uncontrolled study designs are common, allowing selection bias based on clinicians' decision-making. Nevertheless, the studies that used a randomized design also reported inconsistent findings varying from no differences between individuals with and without a co-morbid PD (Hirschfeld et al., 1998; Maddux et al., 2009) to a negative effect of PD

\footnotetext{
* Corresponding author.

E-mail address: suzanne.vanbronswijk@maastrichtuniversity.nl (S.C. van Bronswijk).
} 
on MDD outcome (DeRubeis et al., 2014; Fournier et al., 2008). Another concern is that only a few studies provide data on the relationship between PD and dropout (Kool et al., 2005), which can lead to biased or inaccurate conclusions. The few studies that reported drop-out rates have mixed results: some showed no difference between individuals with and without PD (Fournier et al., 2008; Kool et al., 2003; Sullivan et al., 1994), while others reported higher drop-out rates for individuals with PD (Moradveisi et al., 2013). In addition, despite substantial differences (e.g. depression severity) between individuals with and without PD, multivariable models controlling for significant confounders are rarely used (Casey et al., 2004; Mulder, 2002). Finally, inconsistent findings could also be explained by different outcomes on self-report versus clinician-rated measures of personality pathology and depression outcome (Mulder, 2002; Stanley and Wilson, 2006; Unger et al., 2013).

Concerning the various treatment options for MDD, researchers and clinicians alike have come to realize that a 'one size fits all' approach is not very effective. A major challenge in health care research today is to select the best treatment option for a given individual, a concept referred to as personalized medicine (Simon and Perlis, 2010). Therefore, it is highly relevant to examine whether depressed individuals with comorbid PD respond differentially to evidence-based psychotherapies for MDD. At present, both Cognitive Therapy (CT) and Interpersonal Psychotherapy (IPT) are recommended as first choice evidence-based psychotherapies for MDD (Cuijpers et al., 2014). A recent trial sequential analysis showed that both treatments do not differ in effectiveness for MDD when a difference of 4 BDI-II points is taken as futile (Lemmens et al., 2015).

Previous research comparing CT and IPT head-to-head suggests specific PD features as potential treatment moderators. The National Institute of Mental Health Treatment of Depression Collaborative Program found that depressed individuals with obsessive-compulsive PD features were more likely to respond to IPT, while depressed participants with more avoidant PD features responded better to CT (Barber and Muenz, 1996). These results were partially replicated by the Christchurch Psychotherapy for Depression Study, where individuals with MDD and comorbid PD features responded less well to IPT compared to CT (Carter et al., 2011) particularly in individuals with avoidant PD features (Joyce et al., 2007). In another study, depressive PD features predicted better outcome in CT compared to IPT, while other PD features did not predict differential treatment outcome (Ryder et al., 2010). These inconsistencies might be explained by the use of different PD assessments, which complicates comparison of these findings. Moreover, most studies use simple linear regression models, while multilevel modeling is rarely used. The latter can explore the dynamic and individual course of depression more accurately (De Bolle et al., 2011).

The overall aim of the current study was to determine whether the presence of a DSM PD diagnosis, affected depressive symptom change and treatment retention in CT and IPT during treatment and follow-up. In addition, the effect of PD on remission rates was evaluated, since achieving remission is an evident treatment goal in CT and IPT. Potential differences in these effects between CT and IPT were examined. To acquire a more detailed understanding about cluster C PD features, dimensional scores of obsessive-compulsive, avoidant and dependent PD features as defined by the DSM were calculated, together with cluster A and cluster B PD feature scores. With these scores, general and differential effects of specific PD features on depressive symptom change, treatment retention and remission were examined in CT and IPT. Based on previous studies, we were unsure how PD affected depression outcomes and dropout in CT and IPT during treatment and follow-up. Based on the previous comparisons between CT and IPT we expected cluster $\mathrm{C}$ features, in particular obsessive-compulsive and avoidant PD features to moderate treatment outcomes.

\section{Methods}

\subsection{Design and participants}

Data were collected in the context of a large randomized clinical trial. A detailed description about sample characteristics, study design, interventions, and main treatment outcome findings is provided elsewhere (Lemmens et al., 2011; Lemmens et al., 2015). The study was conducted at the mood disorders unit of the Maastricht Community Mental Health Centre (RIAGG Maastricht) and included 182 outpatients, 18-65 years of age, with a primary diagnosis of MDD (as confirmed with the Structured Clinical Interview for DSM-IV Axis I disorders (SCID-I; First et al., 1995)). Other inclusion criteria were: internet access, an email address, and sufficient knowledge of the Dutch language. Exclusion criteria were a bipolar or chronic (current episode $>5$ years) depression, high acute suicide risk, concomitant pharmacological or psychological treatment, drugs and alcohol abuse/dependence, and mental retardation (IQ < 80).

Participants were randomly allocated to CT $(n=76)$, IPT $(n=75)$, or a Waiting List Condition (WLC; $n=31$ ). For the current analyses, we limited the sample to data of individuals randomized to CT and IPT ( $n=151)$. This sample participated in a treatment phase (0-7 months) and a subsequent trial follow-up phase (7-12 months). Five participants ( 4 in the CT group and 1 in the IPT group) were excluded in the current study, because PD assessments were incomplete or missing. All participants provided written informed consent and the study was approved by the Medical Ethics Committee of Maastricht University Medical Center. The study is registered at The Netherlands Trial Register, part of the Dutch Cochrane Centre (ISRCTN 67561918).

\subsection{Measures}

\subsubsection{Primary outcome}

Primary outcome, depressive symptom severity, was assessed with the Beck Depression Inventory, second edition (BDI-II; Beck et al., 1996). BDI-II measurements included in this study were collected at baseline, at the start of each therapy session and at 3, 7, 8, 9, 10, 11, and 12 months.

\subsubsection{Personality measures}

PD diagnoses were administered prior to treatment using the Structured Clinical Interview for DSM-IV Axis II (SCID-II; First et al., 1997) by well-trained therapists that were participating in the study. In addition, the results of the SCID-II assessment were available in the patient record during treatment. Therefore, therapists and participants were not blind to the results of the SCID-II assessment. Passive-Aggressive PD and Depressive PD were excluded from the analyses, given their position in the section of criteria sets for further study (American Psychiatric Association, 2000). Dimensional PD scores were calculated by summing up the items answered with 'present' for each PD criterion, dismissing items that were scored 'uncertain' or 'absent'. For the cluster C PD features, we calculated individual avoidant PD features scores (range 0-7), dependent PD features scores (range 0-8), and obsessivecompulsive PD features scores (range $0-8$ ). We calculated individual cluster A PD features (range 0-23) and cluster B PD features (range $0-41$ ) total scores providing two reference groups of other, non-Cluster C, PD features.

\subsection{Treatments and therapists}

Both interventions consisted of 16-20 sessions of $45 \mathrm{~min}$, depending on the participants' improvement, with an average of 17 sessions per person ( $S D=2.9$; Lemmens et al., 2015). Sessions were planned weekly and allowed to be less frequently scheduled towards the end of therapy. 
Sample description: demographics, clinical characteristics, quality of life, treatment compliance and depression outcome at 7 and 12 months.

\begin{tabular}{|c|c|c|c|c|}
\hline & \multicolumn{2}{|l|}{ Cognitive Therapy $(n=72)$} & \multicolumn{2}{|c|}{ Interpersonal Psychotherapy $(n=74)$} \\
\hline & $\begin{array}{l}\text { Participants without PD } \\
(n=43)\end{array}$ & $\begin{array}{l}\text { Participants with PD } \\
(n=29)\end{array}$ & $\begin{array}{l}\text { Participants without PD } \\
(n=54)\end{array}$ & $\begin{array}{l}\text { Participants with PD } \\
(n=20)\end{array}$ \\
\hline \multicolumn{5}{|l|}{ Demographics } \\
\hline - Age, years: mean (SD) & $41.7(12.9)$ & $41.2(12.1)$ & $41.5(12.2)$ & $41.0(11.2)$ \\
\hline - Female, $n(\%)$ & $32(74.4)$ & $20(69.0)$ & $35(64.8)$ & $11(55.0)$ \\
\hline - Partner, $n(\%)$ & $23(53.5)$ & $17(58.6)$ & $34(63.0)$ & $16(80.0)$ \\
\hline - Employed, $n$ (\%) & $27(62.8)$ & $16(55.2)$ & $31(57.4)$ & $16(80.0)$ \\
\hline \multicolumn{5}{|l|}{ - Education, $n(\%)$} \\
\hline Low, $n(\%)$ & $8(18.2)$ & $7(25.0)$ & $7(13.0)$ & $6(30.0)$ \\
\hline Medium, $n(\%)$ & $29(67.4)$ & $16(55.2)$ & $30(55.6)$ & $10(50.0)$ \\
\hline High, $n(\%)$ & $6(13.6)$ & $6(21.4)$ & $17(31.5)$ & $4(20.0)$ \\
\hline \multicolumn{5}{|l|}{ Clinical characteristics } \\
\hline - BDI-II baseline score, mean (SD) & $25.9(8.0)^{* *}$ & $32.0(8.7)^{* * k}$ & $30.9(9.3)$ & $32.4(7.7)$ \\
\hline - Recurrent depression, $n(\%)$ & 18 (41.9) & $16(55.2)$ & $24(44.4)$ & $11(55.0)$ \\
\hline - Axis I comorbidity, $n(\%)$ & $15(34.9)$ & $17(58.6)$ & $26(48.2)$ & $12(60.0)$ \\
\hline - BSI, mean (SD) & $56.3(24.8)^{\text {*.t. }}$ & $78.6(30.6)^{* *}$ & $70.3(27.5)$ & $75.2(32.6)$ \\
\hline \multicolumn{5}{|l|}{ Functionality } \\
\hline - WSAS, mean (SD) & $22.1(8.1)$ & $24.4(7.0)$ & $23.1(7.0)$ & $20.8(7.7)$ \\
\hline - EQ-5D, mean (SD) & $0.6(0.2)$ & $0.6(0.2)$ & $0.6(0.3)$ & $0.6(0.3)$ \\
\hline - RAND-36, mean (SD) & $46.7(18.2)$ & $46.8(13.6)$ & $44.3(14.7)$ & $43.8(11.7)$ \\
\hline \multicolumn{5}{|l|}{ Treatment retention } \\
\hline - Dropouts, n (\%) & $12(27.9)$ & $5(17.2)$ & $13(24.1)$ & $2(10.0)$ \\
\hline \multicolumn{5}{|l|}{ Depression outcome: at 7 months } \\
\hline - BDI-II, mean (SD) & $13.6(9.4)$ & $13.3(11.3)$ & $17.1(14.1)$ & $14.3(11.7)$ \\
\hline \multicolumn{5}{|l|}{ Depression outcome: at 12 months } \\
\hline - BDI-II, mean (SD) & $13.1(11.3)$ & $11.2(11.0)$ & $17.9(16.1)$ & $16.3(12.4)$ \\
\hline
\end{tabular}

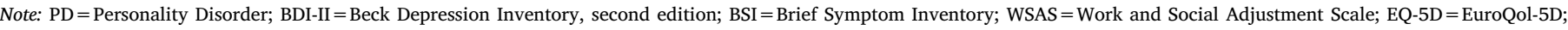
$\mathrm{SD}=$ Standard Deviation.

" $\mathrm{P}<0.05$ differences between participants with PD and participants without PD.

${ }^{* *} \mathrm{P}<0.01$ differences between participants with PD and participants without PD within treatment group.

Interventions were described in CT and IPT protocols following guidelines by Beck et al. (1979) for CT, and guidelines by Klerman et al. (1984) for IPT. Treatments were performed by ten licensed psychologist, psychotherapists, and psychiatrists (five in each condition) with on average 9.1 years $(S D=5.4)$ of clinical experience (range 4-21 years). To avoid treatment contamination, therapist delivered exclusively CT or IPT. All sessions were videotaped. The quality of treatment was rated as good to excellent by independent assessors using the Cognitive Therapy Scale for CT (mean $=3.31, \mathrm{SD}=0.93$; Dobson et al. 1985) and the short version of the IPT Adherence and Quality Scale for IPT (mean $=3.50, \mathrm{SD}=0.70$; Stuart, 2011). There were significant differences in therapy-specific behavior between conditions as indicated by the Collaborative Study Psychotherapy Rating Scale (Hollon et al., 1984, 1988) with higher CT-specific behavior in CT as compared to IPT (mean score of 80.80 v. 52.42, $\mathrm{t}_{79.21}=7.23, \mathrm{p}<0.001$ ), and higher IPTspecific behavior in IPT as compared to CT (85.75 v. 44.57, $t_{86.96}=10.79, p<0.001$; Lemmens et al., 2015). The presence or absence of a comorbid PD diagnosis did not change therapy-specific behavior of the therapists; adherence remained equally well (results not shown, all p-values $>0.3$ ).

\subsection{Statistical analysis}

A detailed description of statistical analyses is presented in online Data Supplement I. Differences in pretreatment (clinical) characteristics and observed mean depression outcomes were examined for individuals with and without PD using descriptive statistics. Study dropout (loss to follow-up) and treatment dropout (discontinuation of the treatment intervention) were compared between individuals with and without PD $(\chi 2)$ and treatment dropout was further investigated with a Cox regression analysis.

Linear (2-level) mixed-effects models were used to examine the effects of PD and PD features on BDI-II change for the treatment phase
(BDI-II scores measured at the start of each therapy session) and the follow-up phase models (BDI-II scores at 7, 8, 9, 10, 11 and 12 months) separately. All linear mixed-effects models included the following fixed effects: BDI-II baseline score (mean centered), a BDI-II baseline score $x$ time interaction, time, treatment (centered at -0.5 for CT, +0.5 for IPT) and a "time $\times$ treatment" interaction. For the time variable, the endpoint was coded as zero (treatment phase model: session 20; trial follow-up phase model: month 12). The effect of PD was analyzed, by adding a predictor of $\mathrm{PD}$ (centered at $-0.5=$ no $\mathrm{PD},+0.5=$ one or more PD's) to the treatment and trial follow-up phase models, with "PD $\times$ time", "PD $\times$ condition" and "PD $\times$ time $\times$ condition" interactions. To analyze the effect of PD features, mean centered scores of avoidant PD features, dependent PD features, obsessive-compulsive PD features, cluster A and cluster B PD features were added simultaneously as continuous predictors to the treatment and trial follow-up phase models with " $\times$ time", " $\times$ condition" and "time $\times$ condition" interactions. To determine the pretreatment associations between PD features and BDIII baseline scores, Pearson correlation coefficients were computed.

To examine effects of PD and PD features on remission, we applied two Cox regression models, with for each a different definition of remission based on the frequency of the BDI-II measurements. For the first model, referred to as the treatment phase Cox regression model, BDI-II scores at the start of each weekly therapy session were used and remission was defined as a BDI-II score of $<10$ for three consecutive weeks. For the second model, referred to as the combined Cox regression model, BDI-II measures at 3, 7, 8, 9, 10, 11 and 12 months across the treatment and trial follow-up phase were used and remission was defined as a BDI-II score $<10$ at one of the assessment points. Dropouts were censored after the last available observation. All models included "condition" as a predictor. The effect of PD was examined by adding PD as a dichotomous predictor (yes/no) to the models, along with a "PD $\times$ condition" interaction. The effect of PD features was examined by adding avoidant PD features, dependent PD features, obsessive- 
compulsive PD features, cluster A PD features, cluster B PD features and their interactions with condition simultaneously to the models.

All outcomes were tested at $\alpha=0.05$ (two-tailed). For all models, we conducted a sensitivity analyses by consecutively adding the following baseline measures to the models: therapist, demographics (age, gender, employment, level of education), clinical features (first or recurrent depression, general psychological distress (BSI; Derogatis and Melisaratos, 1983), axis I comorbidity (SCID-I; First et al., 1995)) and functionality (Work and Social Adjustment Scale (W \& SAS; Mundt et al., 2002), the RAND-36 (van der Zee and Sanderman, 1993) and the EuroQol-6D (EQ-6D; EuroQol, 1990) for the EQ-5D).

\section{Results}

\subsection{Descriptive statistics}

In our sample of 146 participants, 49 individuals were diagnosed with one $(n=37 ; \mathrm{CT}=19$, IPT $=18)$ or multiple PDs $(n=12 ; \mathrm{CT}=10$, IPT $=2$ ). The number of individuals with a PD diagnosis did not significantly differ between the two treatment groups (CT $=29, \mathrm{IPT}=20$, $\chi^{2}=2.87, p=0.09$ ). The most prevalent PD in this sample was an avoidant PD (46.0\%), followed by obsessive-compulsive PD (31.7\%), paranoid PD (6.3\%), borderline PD (6.3\%), dependent PD (3.2\%), schizotypal PD (3.2\%), schizoid Personality Disorder (1.6\%), and PD not otherwise specified (1.6\%). Examining dimensional PD features, participants had on average 1.8 avoidant PD features, 0.9 dependent PD features, 1.5 obsessive-compulsive PD features, 1.3 cluster A PD features and 1.3 cluster B PD features. These PD features did not appear to significantly differ between the two treatments (all $p$ 's $>0.05$ ).

Table 1 provides the differences in baseline demographics, clinical characteristics, functionality measures, treatment compliance, and observed mean treatment outcomes between participants with and without PD in each treatment condition. Baseline BDI-II scores were significantly higher in participants with PD compared to participants without PD $(t(144)=2.28, p=0.02)$. In addition, higher levels of general psychological distress (as measured with the BSI) and more axis I comorbidity were found in participants with PD compared to participants without PD $\left(t(144)=2.61, p=0.01\right.$ and $\chi^{2}(1)=3.73$, $p=0.053)$.

\subsection{The effect of Personality Disorder and PD features on study and treatment dropout}

Participants with and without PD did not differ significantly in proportion of study and treatment dropouts $\left(\chi^{2}(1)=0.27, p=0.60\right.$ and $\left.\chi^{2}(1)=2.51, p=0.113\right)$. When further examining treatment dropout with a Cox regression model, no significant effect of PD was found $(H R=0.59, p=0.33)$ and there was no significant "PD $\mathrm{x}$ treatment interaction" $(H R=0.61, p=0.59)$. Obsessive-compulsive $\mathrm{PD}$ features, dependent PD features, avoidant PD features, cluster A PD features and cluster B PD features did not significantly affect treatment dropout, and their interactions with treatment were not significant (all $p$ 's $>0.3$ ).

\subsection{The effect of Personality Disorder on BDI-II change and remission}

Table 2 summarizes the results of the linear mixed-effects models estimating the effects of PD, "PD $\times$ time", "PD $\times$ condition", and "PD $\times$ time $\times$ condition" on BDI-II change in the treatment and trial followup phase. There was no significant effect of PD on BDI-II change during treatment and trial follow-up phase. Additionally, "PD $\times$ condition" and "PD $\times$ time $\times$ condition" were not significant, indicating no differential effect of PD on depressive symptom change between the two interventions. During the treatment phase, 46 participants achieved remission including 21 participants with PD (43.75\% of all individuals with PD) and 25 participants without PD $(25.51 \%$ of all individuals without PD). No significant effect of PD $(H R=1.5 ; p=0.33)$ or the "PD 


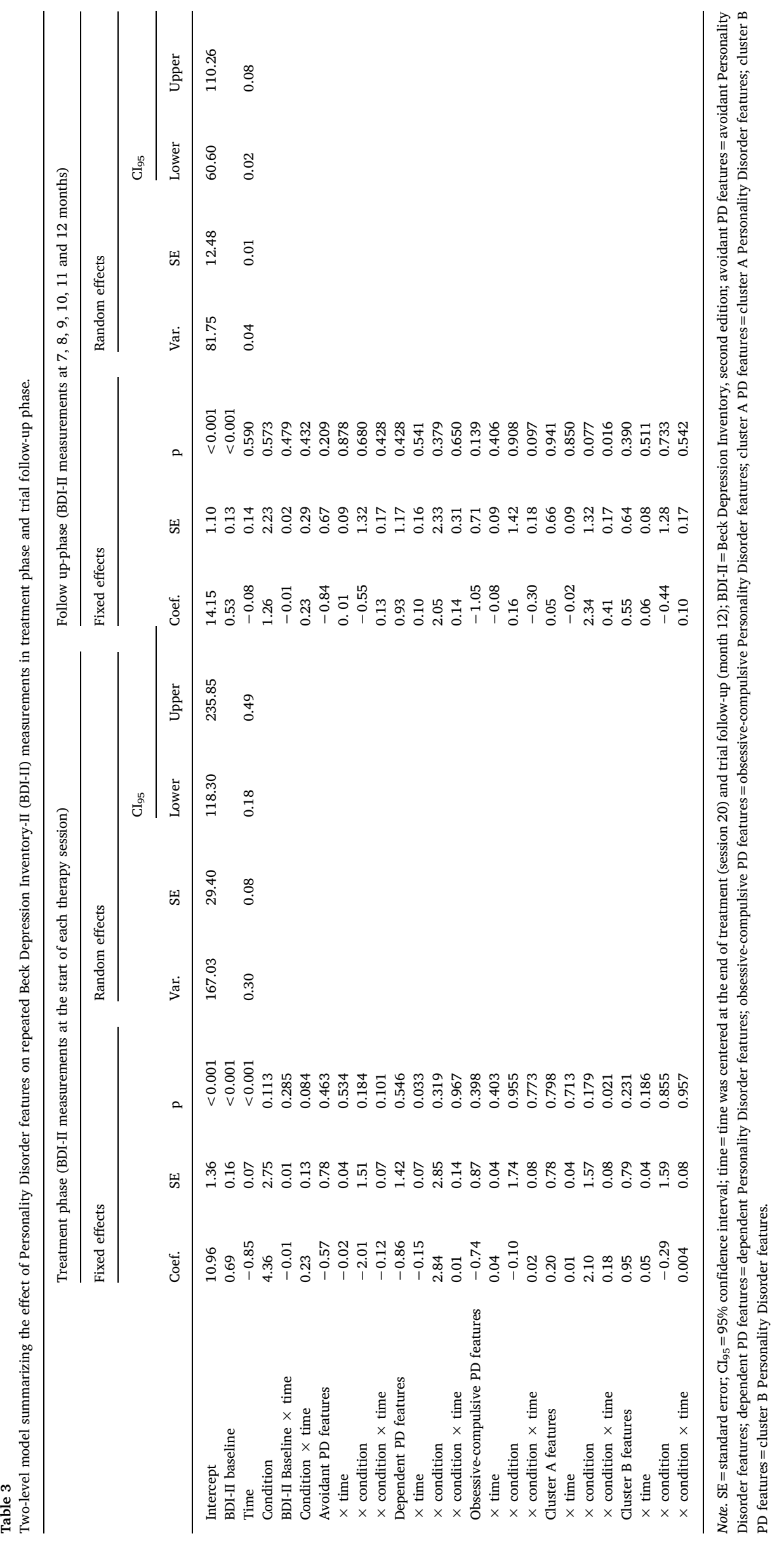


$\times$ condition" interaction $(H R=1.3 ; p=0.63)$ was found with the treatment phase Cox regression model, indicating no (differential) effect of PD on time to remission. In the treatment and trial follow-up phase combined, 74 participants achieved remission, 28 with PD (57.1\% of all individuals with PD) and 46 without PD (47.4\% of all individuals without PD). The combined Cox regression model did not indicate a significant effect of PD on time to remission $(H R=1.1$; $p=0.69$ ), and no significant effect of condition "PD $\times$ condition" $(H R=1.2, p=0.66)$ as well. Sensitivity analyses did not change these results.

\subsection{The effect of Personality Disorder features on BDI-II change and remission}

Table 3 provides estimates of the effects of avoidant PD features, dependent PD features, obsessive-compulsive PD features, cluster A PD features and cluster B PD features and their interactions with " $\times$ time", " $\times$ condition", and " $\times$ time $\times$ condition" on BDI-II change during treatment and follow-up phase. During the treatment phase a significant "dependent PD features $\times$ time" interaction was found, indicating that higher dependent PD features predicted overall lower BDIII scores across the treatment phase. This effect is illustrated in Fig. 1 with plots of the mean BDI-II scores for individuals with high and low dependent features (groups created via a median split). The "dependent

a) Cognitive Therapy

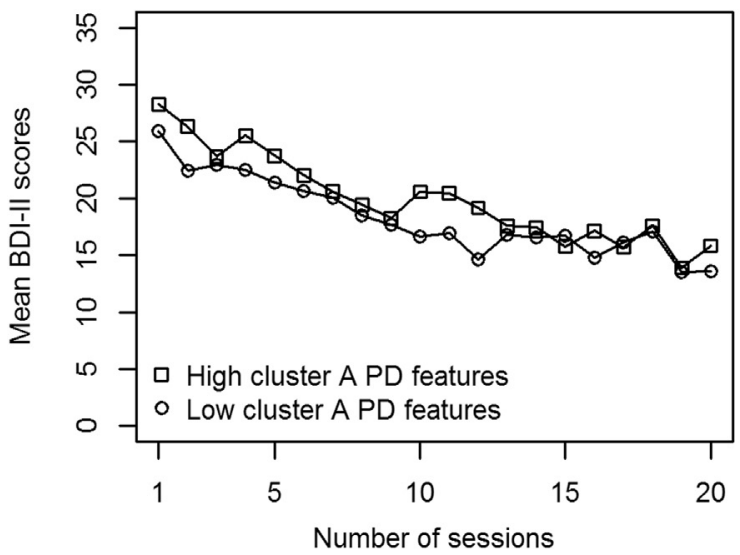

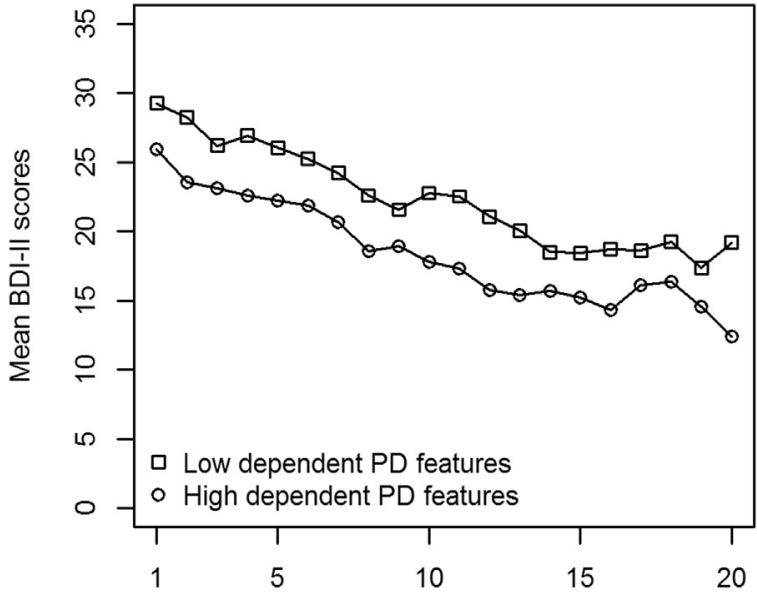

Number of sessions

Fig. 1. Mean Beck Depression Inventory-II (BDI-II) scores for individuals with low and high dependent personality disorder features in the treatment phase. Note: PD: Personality Disorder; Low and high dependent PD features: subgroups of individuals with low and high dependent PD features were created via a medium split.

b) Interpersonal Psychotherapy

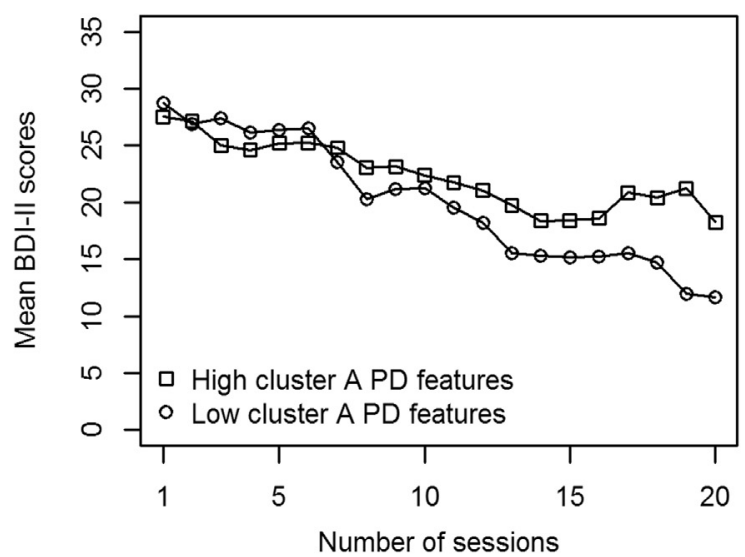

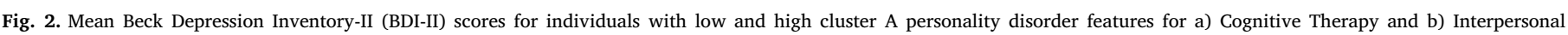

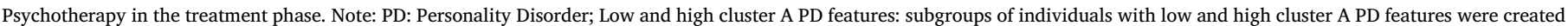
via a medium split.

a) Cognitive Therapy

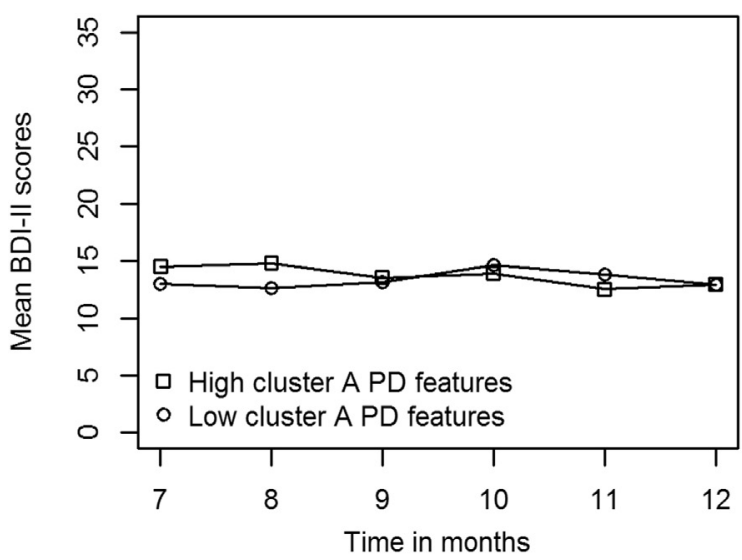

b) Interpersonal Psychotherapy

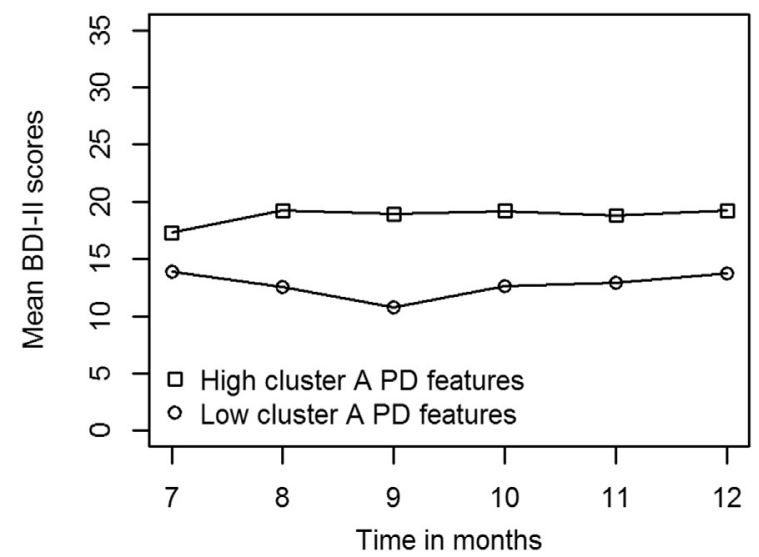

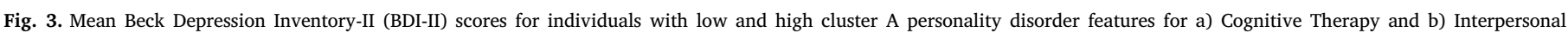

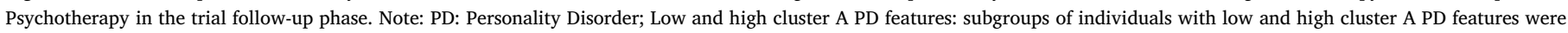
created via a medium split. 
PD features $\times$ time" interaction was no longer significant in the trail follow-up phase. Significant "cluster A PD features $\times$ time $\times$ condition" interactions were found in both the treatment and trial follow-up phase. For IPT, individuals with high cluster A PD features had less BDIII decrease over time as compared to individuals with low cluster A PD features, while cluster A PD features did not affect BDI-II change in CT. This moderating effect is illustrated with plots of mean BDI-II scores for participants with high and low cluster A PD features (groups created via a medium split) in the treatment phase (Fig. 2) and trial follow-up phase (Fig. 3). Obsessive-compulsive PD features, avoidant PD features and cluster B PD features did not show significant (interaction) effects in any of the models. Results were not affected during sensitivity analyses.

The treatment phase Cox regression model indicated that obsessivecompulsive PD features did significantly affect time to remission during the treatment phase $(H R=1.3 ; p=0.006)$ with more OCPD features predicting faster remission for both treatments (no differential effect). However, this effect was not significant in the combined Cox regression model covering both the treatment and trial follow-up phase. Avoidant, dependent, cluster A and cluster B PD features did not affect time to remission in both the treatment phase and the combined Cox regression models and no significant " $\times$ condition" interactions with these feature scores were found. Sensitivity analyses did not change these results.

Pearson correlation coefficients between BDI-II baseline scores and PD features were as follows: avoidant PD features $(r=0.26)$, dependent PD features $(r=0.14)$, obsessive-compulsive PD features $(r=0.10)$, cluster A PD features $(r=0.18)$ and cluster B PD features $(r=0.08)$. Given these weak correlations, it is unlikely that the described PD features effects on BDI-II change were due to pretreatment associations between PD features and depression severity.

\section{Discussion}

The current study examined the impact of PD and PD features on treatment dropout, depressive symptom change and remission in CT and IPT during treatment and a five month follow-up phase. We found that individuals with PD were more severely depressed prior to treatment compared to individuals without PD. Despite these baseline differences, PD (features) did not affect dropout, remission rates and depression severity change for CT and IPT. In addition, more subtle effects were found regarding specific PD features.

As often found in previous studies (Mulder, 2002), individuals with a PD had a more complex clinical picture compared to individuals without a PD prior to treatment, with higher depression severity, higher general psychological distress and more axis I comorbidity. However, PD did not negatively impacted depression severity change and remission rates during treatment and follow-up. Moreover, the percentage of individuals achieving remission was non-significantly higher for participants with PD, and individuals with and without PD had comparable mean post-treatment BDI-II scores, indicative of no adverse effect of PD.

Contrary to what is often assumed, no adverse effects of PD (features) on treatment retention in both CT and IPT were found. This is consistent with most (Fournier et al., 2008; Kool et al., 2003; Sullivan et al., 1994), but not all previous literature: one study reported that PD was associated with higher dropout; however these findings may be somewhat limited, since only a small proportion of the participants had a PD (Moradveisi et al., 2013). Regarding MDD outcome, the current study did not find a (negative) impact of PD on depressive symptom change and remission. With respect to previous head-to-head comparisons of CT and IPT, one study found similar results (Shea et al., 1990), while another study reported an adverse effect of PD on MDD outcome in IPT as compared to CT (Joyce et al., 2007). However, comparing these findings is difficult since one study used another PD assessment instrument and both studies compared pre- and posttreatment depression scores instead of our repeated measurements approach. Based on our findings we suggest that CT and IPT can both attain clinical significant depressive change, irrespective of a more complex clinical presentation at the start of treatment. With regard to the effect of specific PD features, our findings deviate from previous research. Our finding that dependent PD features predicted a BDI-II decrease over time across the treatment phase has not been reported previously. A possible explanation could be that individuals with higher dependent PD features respond initially better to contact with a therapist, but that this effect is lost towards treatment termination. The finding that participants with high cluster A PD features fared better in CT compared to IPT has been previously reported for schizoid symptoms (Joyce et al., 2007). In addition, in a comparison of CT with pharmacotherapy and behavioral therapy, cluster A PD features were associated with better outcomes in CT for the long-term (Moradveisi et al., 2013). However, these effects were absent in the acute phase of MDD. Our findings might be explained by the fact that individuals with higher cluster A PD features respond better to a structured therapy in which the therapists takes a more directive role. In addition, one could argue that focusing on interpersonal problems is quite complex for individuals with cluster A PD features. In previous research, avoidant PD features were found to predict superior treatment outcome in CT compared to IPT (Barber and Muenz, 1996; Joyce et al., 2007), a finding that was not replicated in the current study. The absence of an effect of cluster B PD features on BDI-II change is in line with a study into MDD with comorbid borderline PD of Bellino et al. (2007), although it should be noted that numerous studies have reported otherwise (Agosti et al., 2009; Grilo et al., 2005; Gunderson et al., 2011; Levenson et al., 2012; Skodol et al., 2011). A possible explanation for our finding is that we included participants who met criteria for a primary diagnosis of MDD with comorbid PD, and not participants with a primary PD diagnosis with comorbid MDD.

The finding that comorbid PD did not affect MDD outcome seems at odds with a previous paper using a subset of the same data of this study. This study reported that the presence of any PD predicted lower posttreatment BDI-II scores (Huibers et al., 2015, 2016). These findings derived from a linear regression model with 20 other variables, using a completers sample $(n=134)$, including diagnoses of Passive-Aggressive and Depressive PD. We repeated our treatment phase analyses using the same covariates, data subset and PD diagnoses to explain these conflicting results, but our findings remained similar. However, after removing the random slope (and the autoregressive structure for residuals), a decreasing effect of PD on BDI-II change became apparent but was non-significant $(\beta=-1.97, p=0.11)$. This suggests that the random effects of the multilevel model are responsible for the differences between the analyses, which is in line with our earlier statement that multivariable linear regression approaches are not perfectly suited to address the effects of PD and PD features on treatment outcome reliably. The impact of adding a random slope to our model, underlines the importance of modeling the erratic and individual symptom trajectories of depression.

Contrary to previous findings (De Bolle et al., 2011), our sensitivity analyses did not indicate an effect of therapist differences on treatment outcome. One of the possible reasons for the absence of a therapist effect, may be the (very) good to excellent quality of therapy, and the absence of therapists differences in protocol adherence (Lemmens et al., 2015).

\subsection{Clinical implications}

Translating our findings to clinical practice, a few recommendations can be considered. Firstly, depressed individuals with or without comorbid cluster C PD benefitted equally from both evidence-based psychotherapies and higher premature treatment discontinuation in individuals with personality pathology was not observed. This is relevant as co-morbid cluster C PD is highly prevalent in MDD (Friborg et al., 2014) and research shows that depressed individuals with co-morbid 
PD are less likely to receive adequate MDD treatment (Black et al., 1988; Charney et al., 1981; Lewis and Appleby, 1988). To avoid undertreatment of MDD in individuals with a comorbid cluster C PD, adherence to standard clinical treatment guidelines seems mandatory. Second, based on these findings, depressed individuals with high cluster A PD features could be advised to start with CT instead of IPT, although replication of these results is needed.

\subsection{Methodological considerations}

The current study evaluated the impact of personality pathology on MDD outcome in CT and IPT using state-of-the art multilevel modeling with repeated measurement of depressive symptoms. These models were corrected for baseline BDI-II differences to allow a fair comparison between two initially different participant groups. Other important strengths of this study are the random allocation, the relative large sample size, the low and reported dropout rates and an extensive integrity check (Lemmens et al., 2015) Since these data were collected in an effectiveness study (Lemmens et al., 2015), we can translate our findings more directly to clinical practice, while benefitting from the controlled circumstances.

Our study has some limitations. First, MDD and PD diagnoses were only assessed by one rater, so inter-rater reliability could not be tested. Second, therapist and participants were not blind to the results of the SCID-II assessment. This knowledge could have affected therapeutic strategies and the participant's motivation. Although our adherence check showed no difference in therapy-specific behavior for individuals with and without PD, therapeutic interventions could have been adapted for individuals with PD (e.g. responsivity effects, Stiles, 2009).

Third, dimensional scores of PD features were calculated by summing up the items answered with 'present' for each PD. It can be doubted if every point of increase on these dimensional scales corresponds to a comparable increase of PD severity. Fourth, we based our primary outcome only on one self-report instrument and we did not include a clinician-rated measure of depression severity change. The magnitude of convergence between the two types of measurement is uncertain given different results in the literature (Cuijpers et al., 2010; Vittengl et al., 2013). Fifth, since PD and PD features were only assessed at baseline, we could not determine if these PD findings are state or trait dependent. The PD assessment could be contaminated by the depressive perspective (Fava et al., 2002; Joffe and Regan, 1988). To deal with these complex concepts, a pragmatic approach is suggested: if measures of PD (features) predict (differential) depression outcome when MDD is diagnosed, then they are considered to be useful (Mulder, 2002). Sixth, our primary outcome was the level of depressive symptomatology. It is uncertain if differences in other life domains (e.g., quality of interpersonal relations) would emerge between remitted individuals with our without PD after successful treatment. Finally, since appointments were allowed to be scheduled in a flexible manner some participants received one or two more therapy sessions during the trial follow-up phase, but this is unlikely to impact our outcomes drastically.

\subsection{Future directions}

More research is needed to further explore our results. Since our sample, in line with previous outpatient research (Friborg et al., 2014), consisted predominantly of individuals with a cluster $\mathrm{C}$ diagnosis, our considerations about cluster A and B PDs on MDD outcome in IPT and CT should be interpreted with caution. Although our study demonstrates promising results of cluster A PD features as potential treatment moderators, further research is needed to replicate these findings. Since cluster A PD features is a heterogeneous category, a more detailed understanding about specific cluster A PD features is warranted. Ideally, a 'what fits for whom' algorithm, based on individual personality features can be generated. While this study focused on the treatment phase and a five-month trial follow-up phase, a number of studies indicate an adverse effect of PD on long-term MDD outcomes (Grilo et al., 2010; Markowitz et al., 2007). Further research is needed to examine the enduring effects of PD and PD features on CT and IPT outcomes over time. Preferably, when examining long-term outcome effects, PD and MDD should be simultaneously and frequently assessed to determine state and trait dependent changes.

\section{Funding}

This research was funded by the Research Institute of Experimental Psychopathology (EPP), the Netherlands, and the Academic Community Mental Health Centre (RIAGG, now Virenze-RIAGG Maastricht) in Maastricht, the Netherlands. Both institutes had no involvement in the collection, analysis and interpretation of the data or in the writing of this article and decision to submit this article for publication.

\section{Acknowledgements}

We would like to acknowledge the contribution of participants and therapists at RIAGG Maastricht. Furthermore, we thank Annie Raven and Annie Hendriks for their assistance during the study.

\section{Appendix A. Supporting information}

Supplementary data associated with this article can be found in the online version at http://dx.doi.org/10.1016/j.jad.2017.08.043.

\section{References}

Agosti, V., Hellerstein, D.J., Stewart, J.W., 2009. Does personality disorder decrease the likelihood of remission in early-onset chronic depression? Compr. Psychiatry 50, 491-495.

American Psychiatric Association, 2000. Diagnostic and Statistical Manual of Mental Disorders (4th ed.). Washington DC.

Barber, J.P., Muenz, L.R., 1996. The role of avoidance and obsessiveness in matching patients to cognitive and interpersonal psychotherapy: empirical findings from the treatment for depression collaborative research program. J. Consult. Clin. Psychol. 64, 951-958.

Beck, A.T., Rush, A.J., Shaw, B.F., Emery, G., 1979. Cognitive Therapy of Depression. Guilford Press, New York.

Beck, A.T., Steer, R., Brown, G.K., 1996. Beck Depression Inventory II: Manual. Harcourt Brace, Boston.

Bellino, S., Zizza, M., Rinaldi, C., Bogetto, F., 2007. Combined therapy of major depression with concomitant borderline personality disorder: comparison of interpersonal and cognitive psychotherapy. Can. J. Psychiatry 52 (11), 718-725.

Black, D.W., Bell, S., Hulbert, J., Nasrallah, A., 1988. The importance of Axis II in patients with major depression. A controlled study. J. Affect. Disord. 14, 115-122.

Carter, J.D., Luty, S.E., McKenzie, J.M., Mulder, R.T., Frampton, C.M., Joyce, P.R., 2011 Patient predictors of response to cognitive behaviour therapy and interpersonal psychotherapy in a randomised clinical trial for depression. J. Affect. Disord. 128, 252-261.

Casey, P., Birbeck, G., McDonagh, C., Horgan, A., Dowrick, C., Dalgard, O., Lethinen, V., Ayuso-Mateos, J.L., Dunn, G., Page, H., Wilkinson, C., Wilkinson, G., VazquezBarquero, J.L., Group, O., 2004. Personality disorder, depression and functioning: results from the ODIN study. J. Affect. Disord. 82, 277-283.

Charney, D.S., Nelson, J.C., Quinlan, D.M., 1981. Personality traits and disorder in depression. Am. J. Psychiatry 138, 1601-1604.

Cuijpers, P., Li, J., Hofmann, S.G., Andersson, G., 2010. Self-reported versus clinicianrated symptoms of depression as outcome measures in psychotherapy research on depression: a meta-analysis. Clin. Psychol. Rev. 30, 768-778.

Cuijpers, P., Karyotaki, E., Weitz, E., Andersson, G., Hollon, S.D., van Straten, A., 2014. The effects of psychotherapies for major depression in adults on remission, recovery and improvement: a meta-analysis. J. Affect. Disord. 159, 118-126.

De Bolle, M., De Fruyt, F., Quilty, L.C., Rolland, J.P., Decuyper, M., Bagby, R.M., 2011. Does personality disorder co-morbidity impact treatment outcome for patients with major depression? A multi-level analysis. J. Pers. Disord. 25, 1-15.

Derogatis, L.R., Melisaratos, N., 1983. The Brief Symptom Inventory: an introductory report. Psychol. Med 13, 595-605.

DeRubeis, R.J., Cohen, Z.D., Forand, N.R., Fournier, J.C., Gelfand, L.A., Lorenzo-Luaces, L., 2014. The Personalized Advantage Index: translating research on prediction into individualized treatment recommendations. A demonstration. PLoS One 9, e83875.

Dobson, K.S., Shaw, B.F., Vallis, T.M., 1985. Reliability of a measure of the quality of cognitive therapy. Br. J. Clin. Psychol. 24, 295-300.

EuroQol, G., 1990. EuroQol-a new facility for the measurement of health-related quality of life. Health Policy 16, 199-208.

Fava, M., Farabaugh, A.H., Sickinger, A.H., Wright, E., Alpert, J.E., Sonawalla, S., Nierenberg, A.A., Worthington 3rd, J.J., 2002. Personality disorders and depression. 
Psychol. Med. 32, 1049-1057.

First, M.B., Spitzer, R.L., Gibbon, M., Williams, J.B.W., 1995. Structured Clinical Interview for DSM-IV Axis I Disorders (SCID-I). Biometrics Research Department New York State Psychiatric Institute, New York.

First, M.B., Gibbon, M., Spitzer, R.L., Williams, J.B.W., Benjamin, L.S., 1997. Structured Clinical Interview for DSM-IV Axis II Personality Disorders (SCID-II). American Psychiatric Press, Washington, D.C.

Fournier, J.C., DeRubeis, R.J., Shelton, R.C., Gallop, R., Amsterdam, J.D., Hollon, S.D., 2008. Antidepressant medications v. cognitive therapy in people with depression with or without personality disorder. Br. J. Psychiatry 192, 124-129.

Friborg, O., Martinsen, E.W., Martinussen, M., Kaiser, S., Overgard, K.T., Rosenvinge, J.H., 2014. Comorbidity of personality disorders in mood disorders: a meta-analytic review of 122 studies from 1988 to 2010. J. Affect. Disord. 152-154, 1-11.

Grilo, C.M., Sanislow, C.A., Shea, M.T., Skodol, A.E., Stout, R.L., Gunderson, J.G., Yen, S., Bender, D.S., Pagano, M.E., Zanarini, M.C., 2005. Two-year prospective naturalistic study of remission from major depressive disorder as a function of personality disorder comorbidity. J. Consult. Clin. Psychol. 73, 78.

Grilo, C.M., Stout, R.L., Markowitz, J.C., Sanislow, C.A., Ansell, E.B., Skodol, A.E., Bender, D.S., Pinto, A., Shea, M.T., Yen, S., Gunderson, J.G., Morey, L.C., Hopwood, C.J., McGlashan, T.H., 2010. Personality disorders predict relapse after remission from an episode of major depressive disorder: a 6-year prospective study. J. Clin. Psychiatry $71,1629-1635$.

Gunderson, J.G., Stout, R.L., McGlashan, T.H., Shea, M.T., Morey, L.C., Grilo, C.M., Zanarini, M.C., Yen, S., Markowitz, J.C., Sanislow, C., 2011. Ten-year course of borderline personality disorder: psychopathology and function from the Collaborative Longitudinal Personality Disorders study. Arch. Gen. Psychiatry 68, 827-837.

Hardy, G.E., Barkham, M., Shapiro, D.A., Stiles, W.B., Rees, A., Reynolds, S., 1995. Impact of Cluster C personality disorders on outcomes of contrasting brief psychotherapies for depression. J. Consult. Clin. Psychol. 63, 997-1004.

Hirschfeld, R.M., Russell, J.M., Delgado, P.L., Fawcett, J., Friedman, R.A., Harrison, W.M., Koran, L.M., Miller, I.W., Thase, M.E., Howland, R.H., Connolly, M.A., Miceli, R.J., 1998. Predictors of response to acute treatment of chronic and double depression with sertraline or imipramine. J. Clin. Psychiatry 59, 669-675.

Hollon, S.D., Waskow, I.E., Evans, M., Lowery, H.A., 1984. Systems for Rating Therapies for Depression. Annual Convention of the American Psychiatric Association, Los Angeles, CA.

Hollon, S.D., Evans, M.D., Auerbach, A., DeRubeis, R.J., Elkin, I.,Lowery, A., Kriss, M., Grove, W., Tuason, V.B., Piasecki, J., 1988. Development of a system for rating therapies for depression: differentiating cognitive therapy, interpersonal psychotherapy, and clinical management pharmacotherapy (unpublished manuscript).

Huibers, M.J., Cohen, Z.D., Lemmens, L.H., Arntz, A., Peeters, F.P., Cuijpers, P., DeRubeis, R.J., 2015. Predicting optimal outcomes in cognitive therapy or interpersonal psychotherapy for depressed individuals using the personalized advantage index approach. PLoS One 10, e0140771.

Huibers, M.J., Cohen, Z.D., Lemmens, L.H., Arntz, A., Peeters, F.P., Cuijpers, P., DeRubeis, R.J., 2016. Correction: predicting optimal outcomes in cognitive therapy or interpersonal psychotherapy for depressed individuals using the personalized advantage index approach. PLoS One 11.

Joffe, R.T., Regan, J.J., 1988. Personality and depression. J. Psychiatr. Res. 22, 279-286.

Joyce, P.R., McKenzie, J.M., Carter, J.D., Rae, A.M., Luty, S.E., Frampton, C.M., Mulder, R.T., 2007. Temperament, character and personality disorders as predictors of response to interpersonal psychotherapy and cognitive-behavioural therapy for depression. Br. J. Psychiatry 190, 503-508.

Klerman, G.L., Weissman, M.M., Rounsaville, B.J., Chevron, E.S., 1984. Interpersonal Psychotherapy for Depression. Basis Books, New York.

Kool, S., Dekker, J., Duijsens, I.J., de Jonghe, F., Puite, B., 2003. Efficacy of combined therapy and pharmacotherapy for depressed patients with or without personality disorders. Harv. Rev. Psychiatry 11, 133-141.

Kool, S., Schoevers, R., de Maat, S., Van, R., Molenaar, P., Vink, A., Dekker, J., 2005. Efficacy of pharmacotherapy in depressed patients with and without personality disorders: a systematic review and meta-analysis. J. Affect. Disord. 88, 269-278.

Lemmens, L.H.J.M., Arntz, A., Peeters, F., Hollon, S.D., Roefs, A., Huibers, M.J., 2011.
Effectiveness, relapse prevention and mechanisms of change of cognitive therapy vs. interpersonal therapy for depression: study protocol for a randomised controlled trial. Trials 12,150

Lemmens, L.H.J.M., Arntz, A., Peeters, F., Hollon, S.D., Roefs, A., Huibers, M.J., 2015 Clinical effectiveness of cognitive therapy v. interpersonal psychotherapy for depression: results of a randomized controlled trial. Psychol. Med. 1-16.

Levenson, J.C., Wallace, M.L., Fournier, J.C., Rucci, P., Frank, E., 2012. The role of personality pathology in depression treatment outcome with psychotherapy and pharmacotherapy. J. Consult. Clin. Psychol. 80, 719.

Lewis, G., Appleby, L., 1988. Personality disorder: the patients psychiatrists dislike. Br. J. Psychiatry 153, 44-49.

Maddux, R.E., Riso, L.P., Klein, D.N., Markowitz, J.C., Rothbaum, B.O., Arnow, B.A., Manber, R., Blalock, J.A., Keitner, G.I., Thase, M.E., 2009. Select comorbid personality disorders and the treatment of chronic depression with nefazodone, targeted psychotherapy, or their combination. J. Affect. Disord. 117, 174-179.

Markowitz, J.C., Skodol, A.E., Petkova, E., Cheng, J., Sanislow, C.A., Grilo, C.M., Gunderson, J.G., McGlashan, T.H., 2007. Longitudinal effects of personality disorders on psychosocial functioning of patients with major depressive disorder. J. Clin. Psychiatry 68, 186-193.

Moradveisi, L., Huibers, M.J., Renner, F., Arasteh, M., Arntz, A., 2013. The influence of comorbid personality disorder on the effects of behavioural activation vs. antidepressant medication for major depressive disorder: results from a randomized trial in Iran. Behav. Res. Ther. 51, 499-506.

Mulder, R.T., 2002. Personality pathology and treatment outcome in major depression: a review. Am. J. Psychiatry 159, 359-371.

Mundt, J.C., Marks, I.M., Shear, M.K., Greist, J.H., 2002. The Work and Social Adjustment Scale: a simple measure of impairment in functioning. Br. J. Psychiatry 180 , $461-464$.

Newton-Howes, G., Tyrer, P., Johnson, T., Mulder, R., Kool, S., Dekker, J., Schoevers, R., 2014. Influence of personality on the outcome of treatment in depression: systematic review and meta-analysis. J. Pers. Disord. 28, 577-593.

Reich, J.H., Vasile, R.G., 1993. Effect of personality disorders on the treatment outcome of axis I conditions: an update. J. Nerv. Ment. Dis. 181, 475-484.

Ryder, A.G., Quilty, L.C., Vachon, D.D., Bagby, R.M., 2010. Depressive personality and treatment outcome in major depressive disorder. J. Pers. Disord. 24, 392-404.

Sato, T., Sakado, K., Sato, S., Morikawa, T., 1994. Cluster a personality disorder: a marker of worse treatment outcome of major depression? Psychiatry Res. 53, 153-159.

Shea, M.T., Pilkonis, P.A., Beckham, E., Collins, J.F., Elkin, I., Sotsky, S.M., Docherty, J.P., 1990. Personality disorders and treatment outcome in the NIMH Treatment of Depression Collaborative Research Program. Am. J. Psychiatry 147, 711-718.

Simon, G.E., Perlis, R.H., 2010. Personalized medicine for depression: can we match patients with treatments? Am. J. Psychiatry 167, 1445-1455.

Skodol, A.E., Grilo, C.M., Keyes, K.M., Geier, T., Grant, B.F., Hasin, D.S., 2011. Relationship of personality disorders to the course of major depressive disorder in a nationally representative sample. Am. J. Psychiatry 168, 257-264.

Stanley, B., Wilson, S.T., 2006. Heightened subjective experience of depression in borderline personality disorder. J. Personal. Disord. 20, 307-318.

Stiles, W.B., 2009. Responsiveness as an obstacle for psychotherapy outcome research: it's worse than you think. Clin. Psychol.: Sci. Pract. 16, 86-91.

Stuart, S., 2011. IPT Adherence and Quality Scale. Interpersonal Psychotherapy Institute, Iowa (unpublished manuscript).

Sullivan, P.F., Joyce, P.R., Mulder, R.T., 1994. Borderline personality disorder in major depression. J. Nerv. Ment. Dis. 182, 508-516.

Unger, T., Hoffmann, S., Köhler, S., Mackert, A., Fydrich, T., 2013. Personality disorders and outcome of inpatient treatment for depression: a 1-year prospective follow-up study. J. Personal. Disord. 27, 636-651.

van der Zee, K.I., Sanderman, R., 1993. Het meten van de algemene gezondheidstoestand met de RAND-36: een handleiding. Noordelijk Centrum voor Gezondheidsvraagstukken, Groningen.

Vittengl, J.R., Clark, L.A., Thase, M.E., Jarrett, R.B., 2013. Nomothetic and idiographic symptom change trajectories in acute-phase cognitive therapy for recurrent depression. J. Consult. Clin. Psychol. 81, 615. 